\title{
Optimized DNA Extracting Method for Oxford Nanopore- Long reads Sequencing from Marine samples
}

\author{
Sonia Boughattas ${ }^{1}$, Dana Albatesh ${ }^{1}$, Bruno W. Giraldes ${ }^{2}$, Asmaa A. Althani ${ }^{1}$, Fatiha M. Benslimane ${ }^{1 *}$ \\ ${ }^{1}$ Biomedical Research Center, ${ }^{2}$ Enivromental science Center - Qatar University - Doha, Qatar. "Correspondence: fatiha@qu.edu.qa
}

\section{Background}

Qatar's particular geographic location represents a marine barrier for different species that have migrated from the neighboring environments. Due to the extreme temperatures and salinities in the gulf region, the national biodiversity has adapted to survive under extreme conditions. Furthermore, the barriers that isolates the Arabian Gulf has created an environment that is rich with endemic species that are specific to the region. The aims of our project is to identify the potential new marine species at Qatar Marine Zone trough whole genome sequencing using Oxford Nanopore Technology (ONT). DNA extracted from marine samples is known to be particularly challenging to sequence due to unknown reasons. Therefore, we surveyed a wide range of genomic DNA extraction protocols to assess their efficiency in providing high quality DNA that is suitable for long read sequencing.

\section{Methods and Results}

Samples containing two worms specimen (Spirobranchus $s p$.) were subjected to several extraction protocols. They were then sequenced on Gridion using ligation kit SQK-LSK109. We investigated liquid nitrogen LqN (few minutes) and mechanical homogenization (Homg) for 10sec twice as a pre-treatment step.

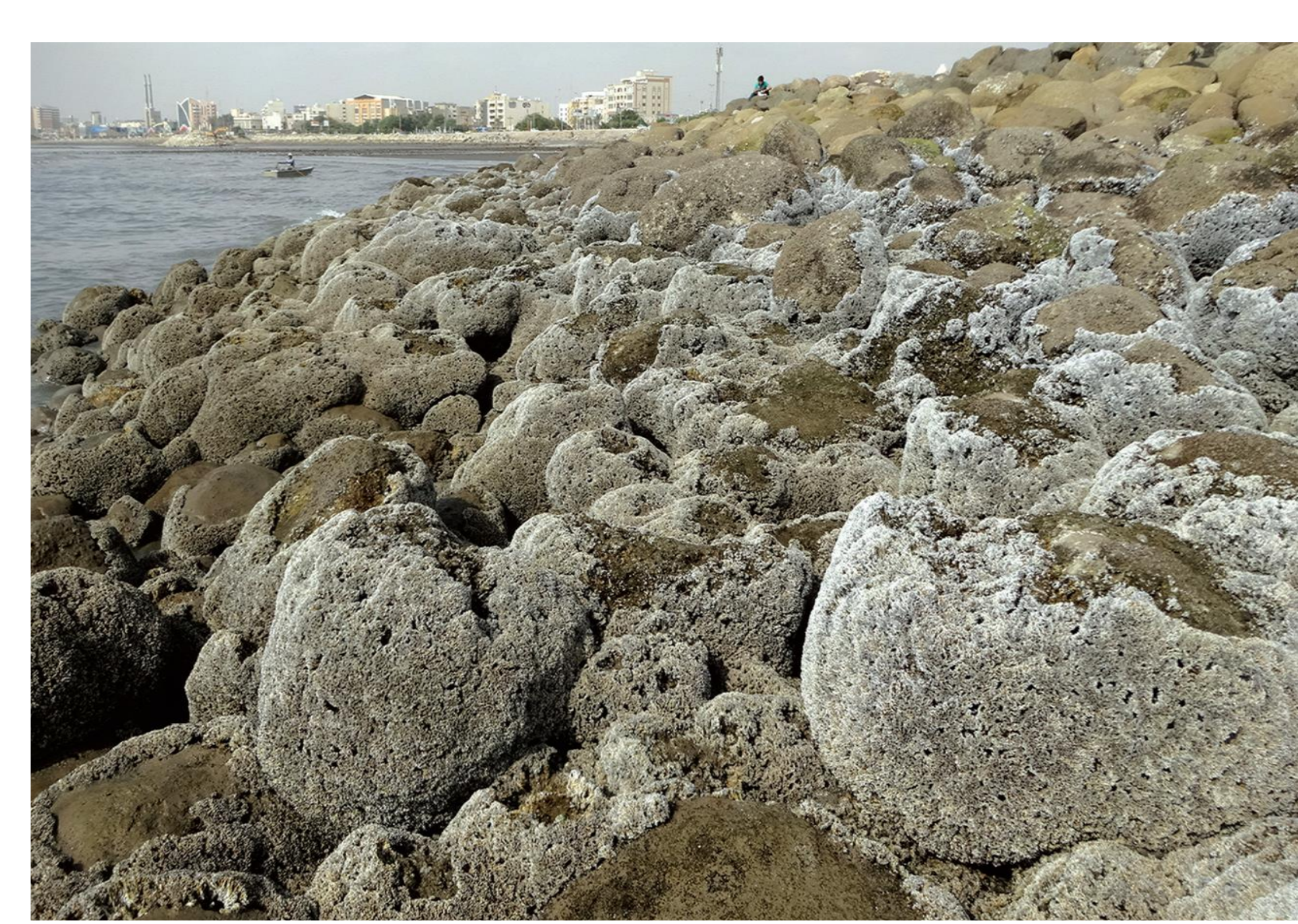

Spirobranchus Sp.

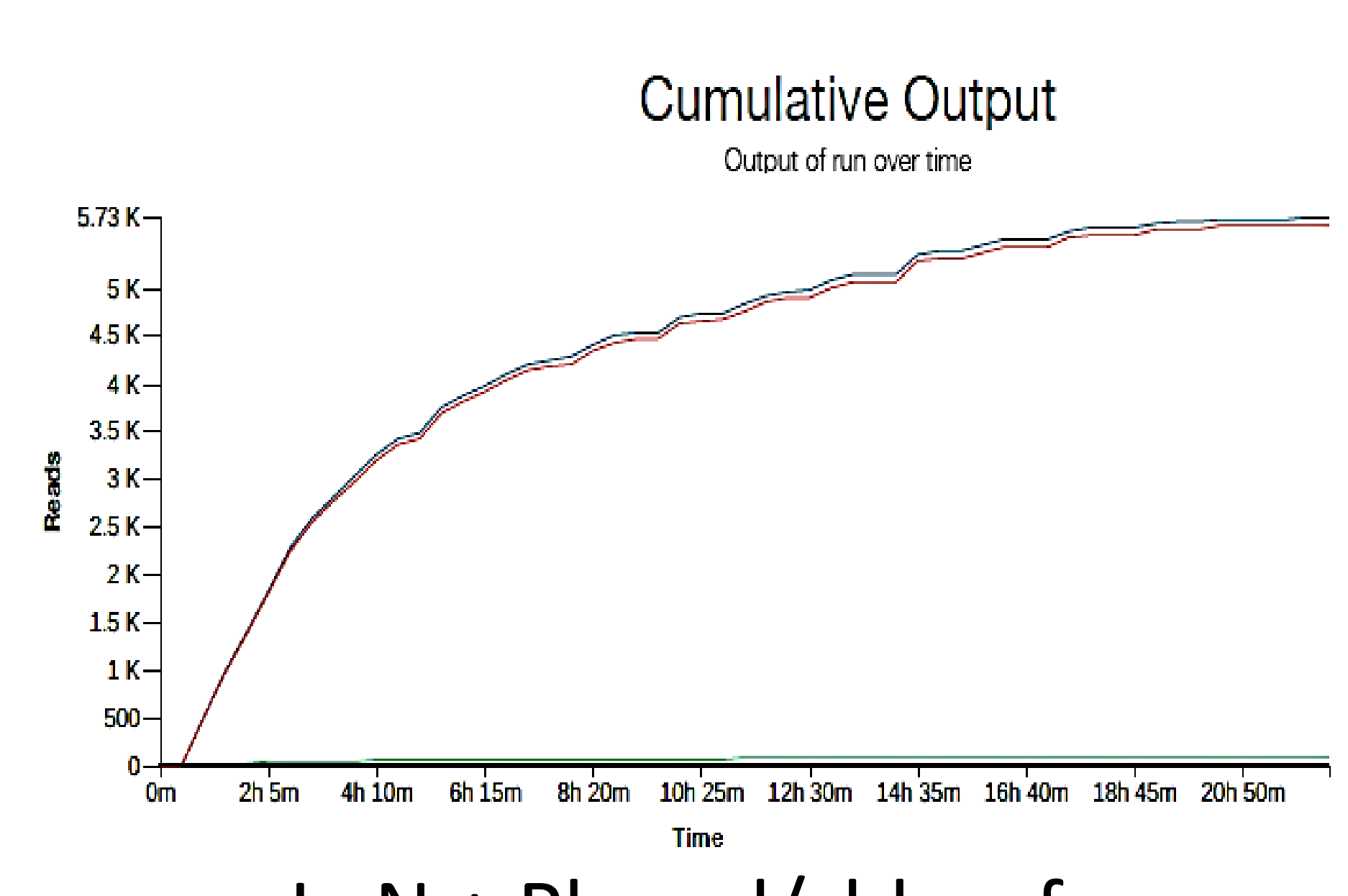

LqN + Phenol/chlorofom

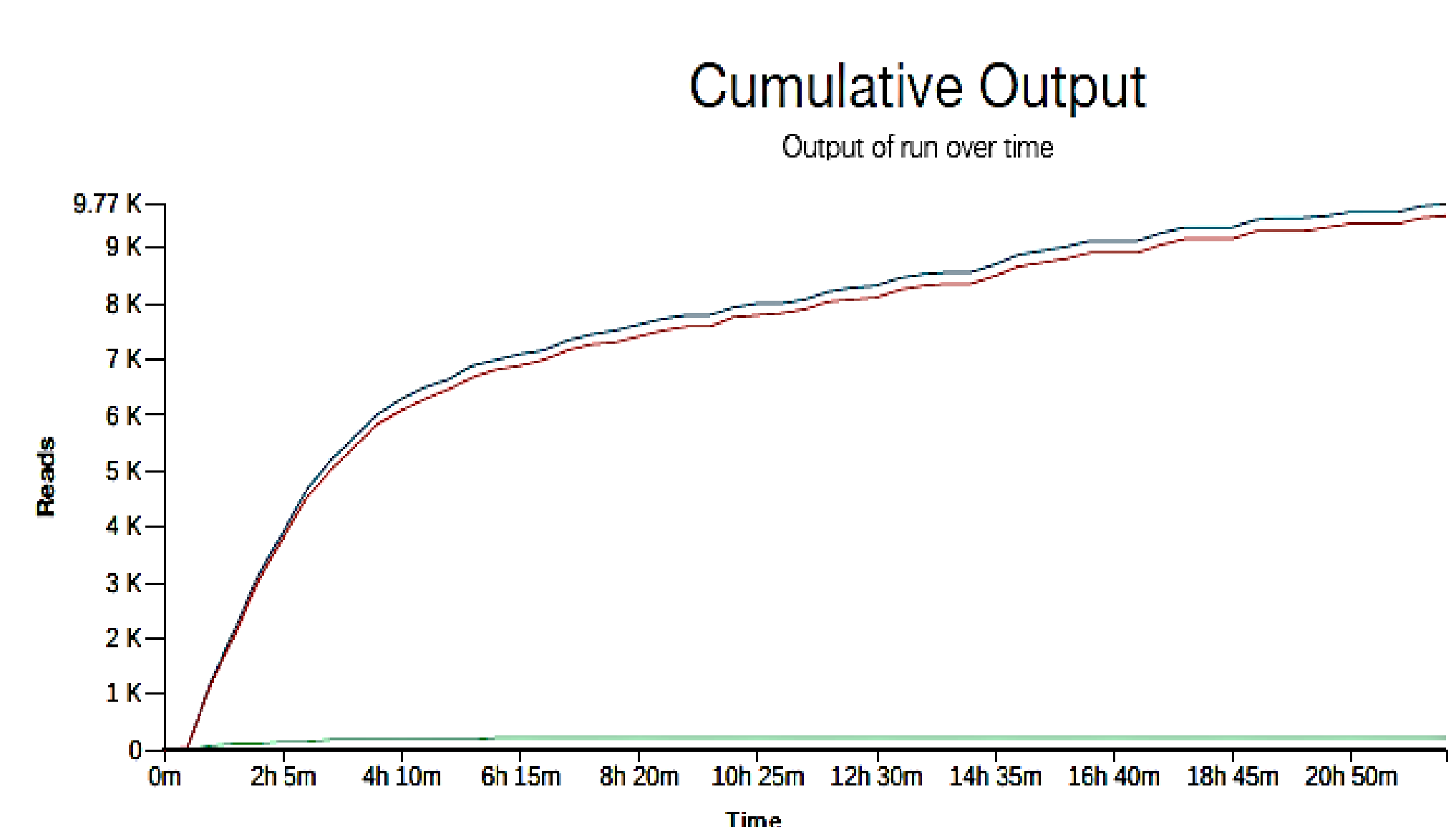

LqN + Genomic Tips

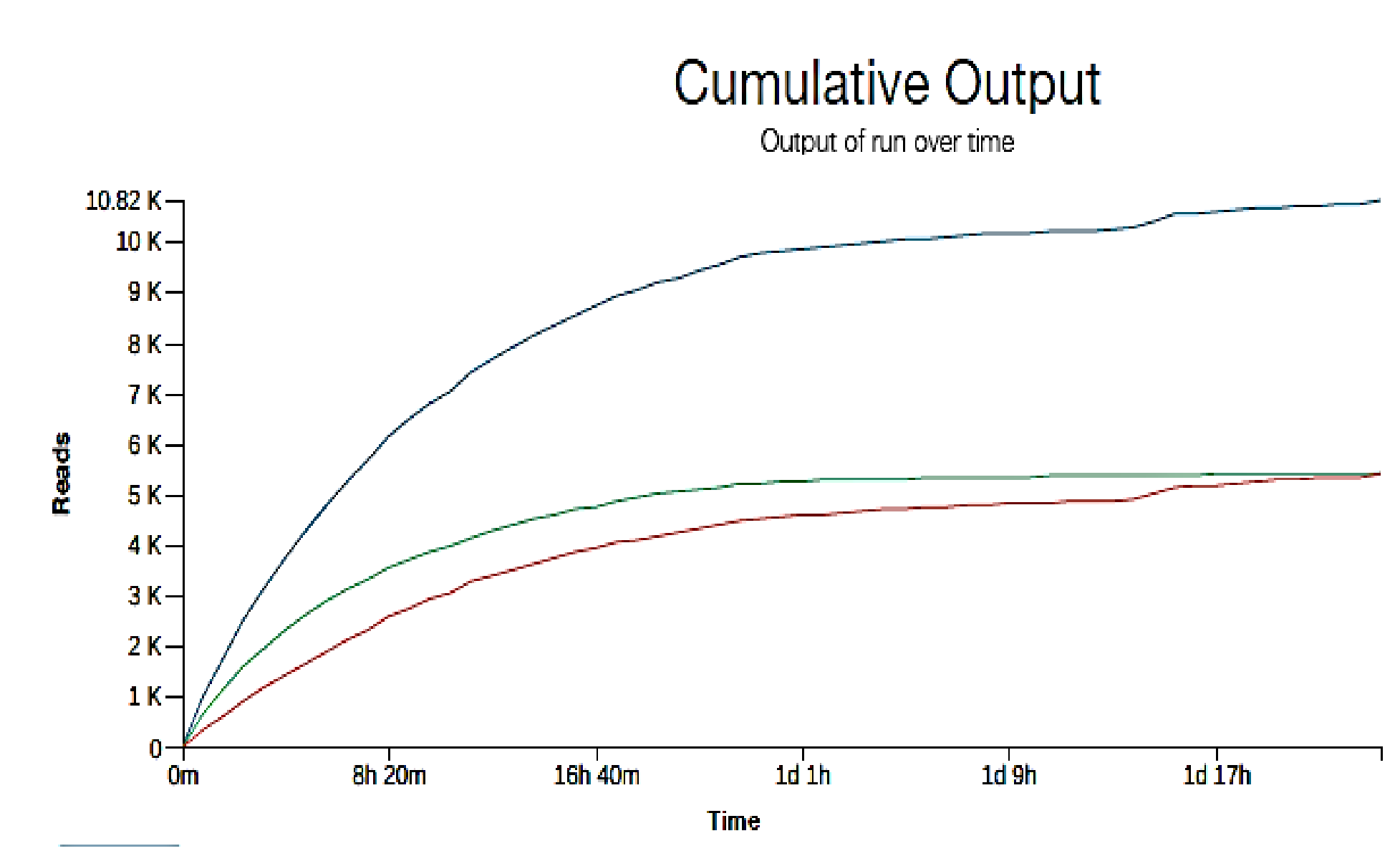

Homg + Qiagen Dneasy Plant Kit
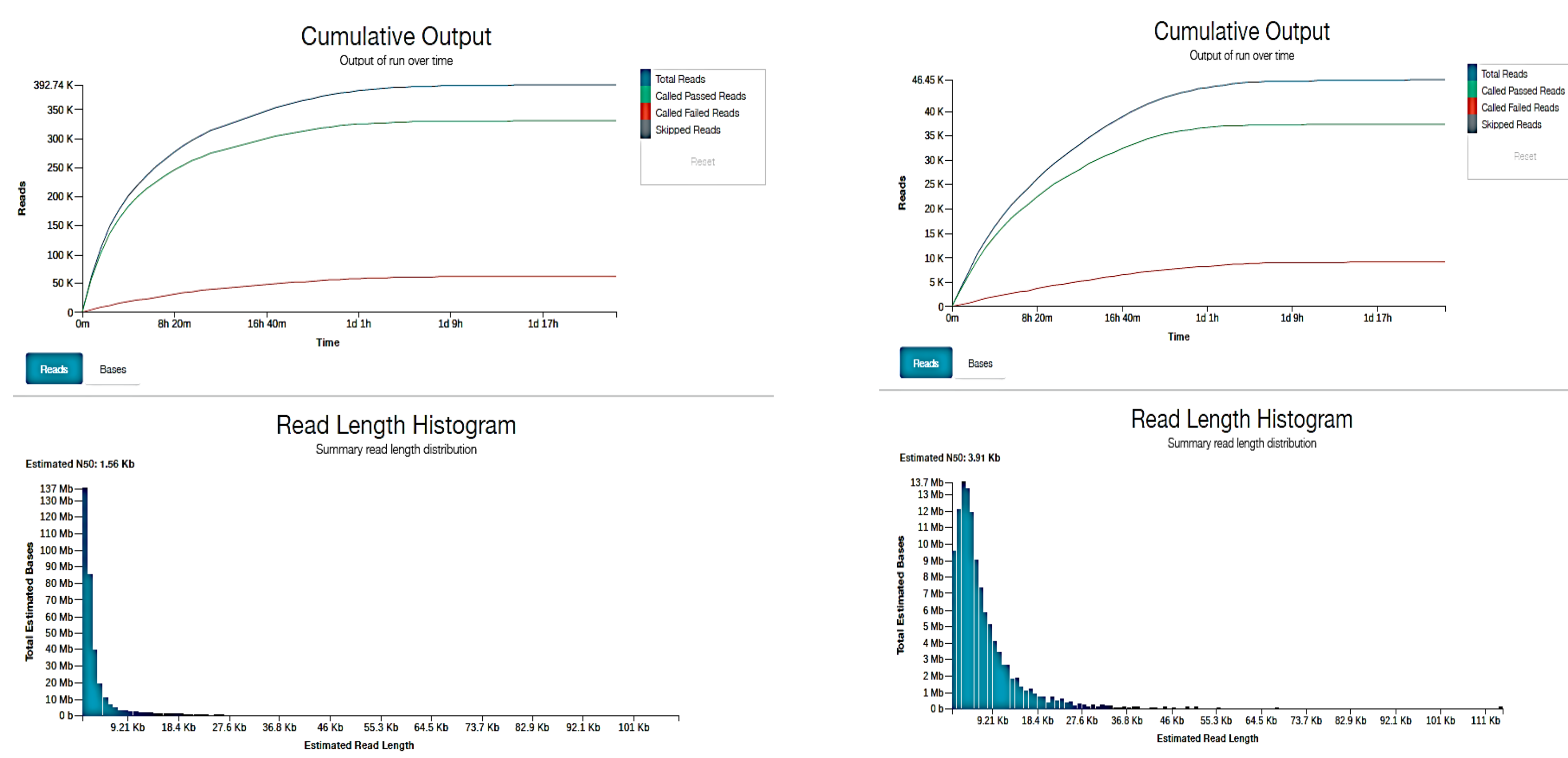

Homg + Qiagen QIAmp DNA Stool Kit
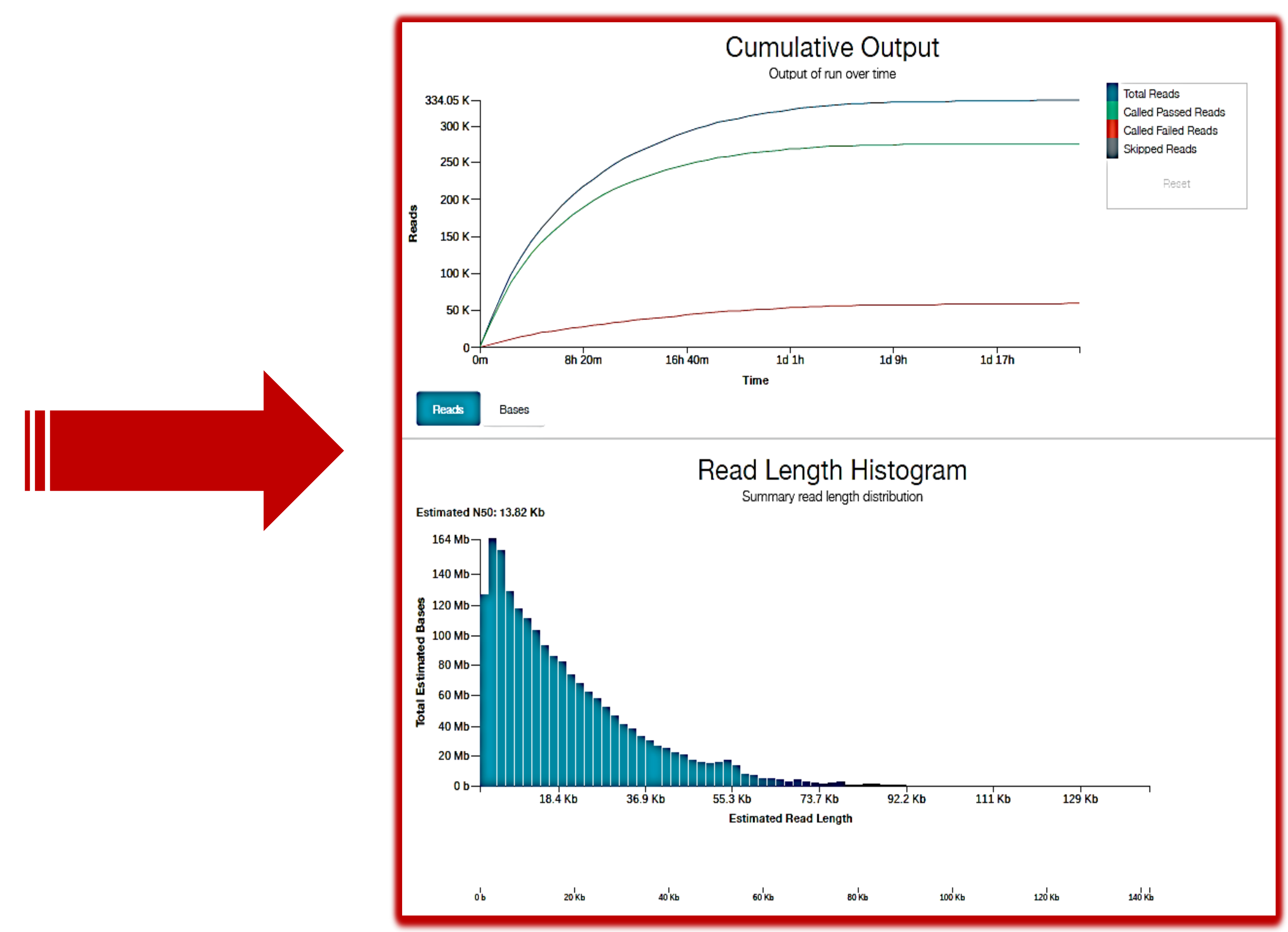

Homg + Bespoke protocol

Homg + Qiagen Dneasy Tissue Kit

Palaemon Sp.

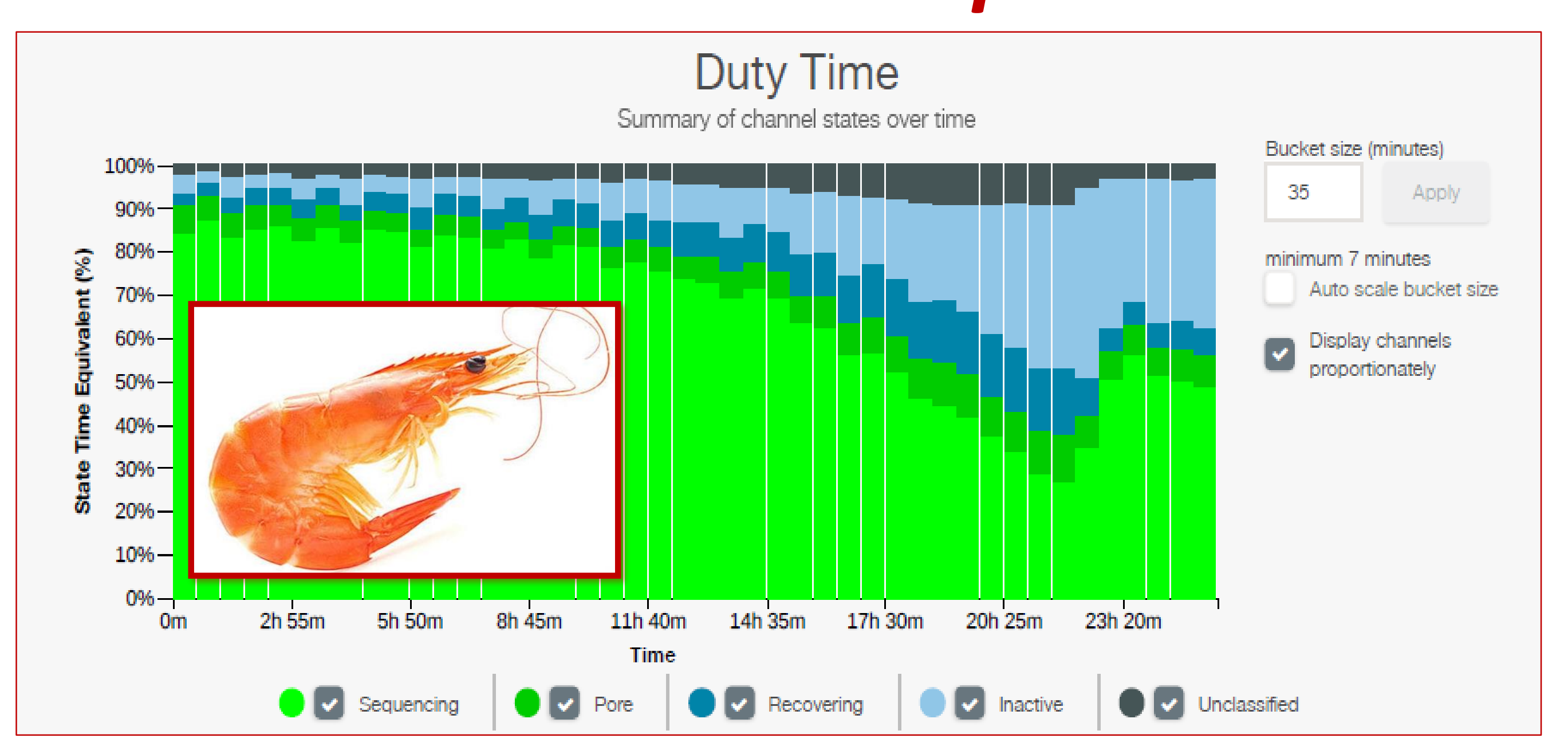

Chrysaora Sp.

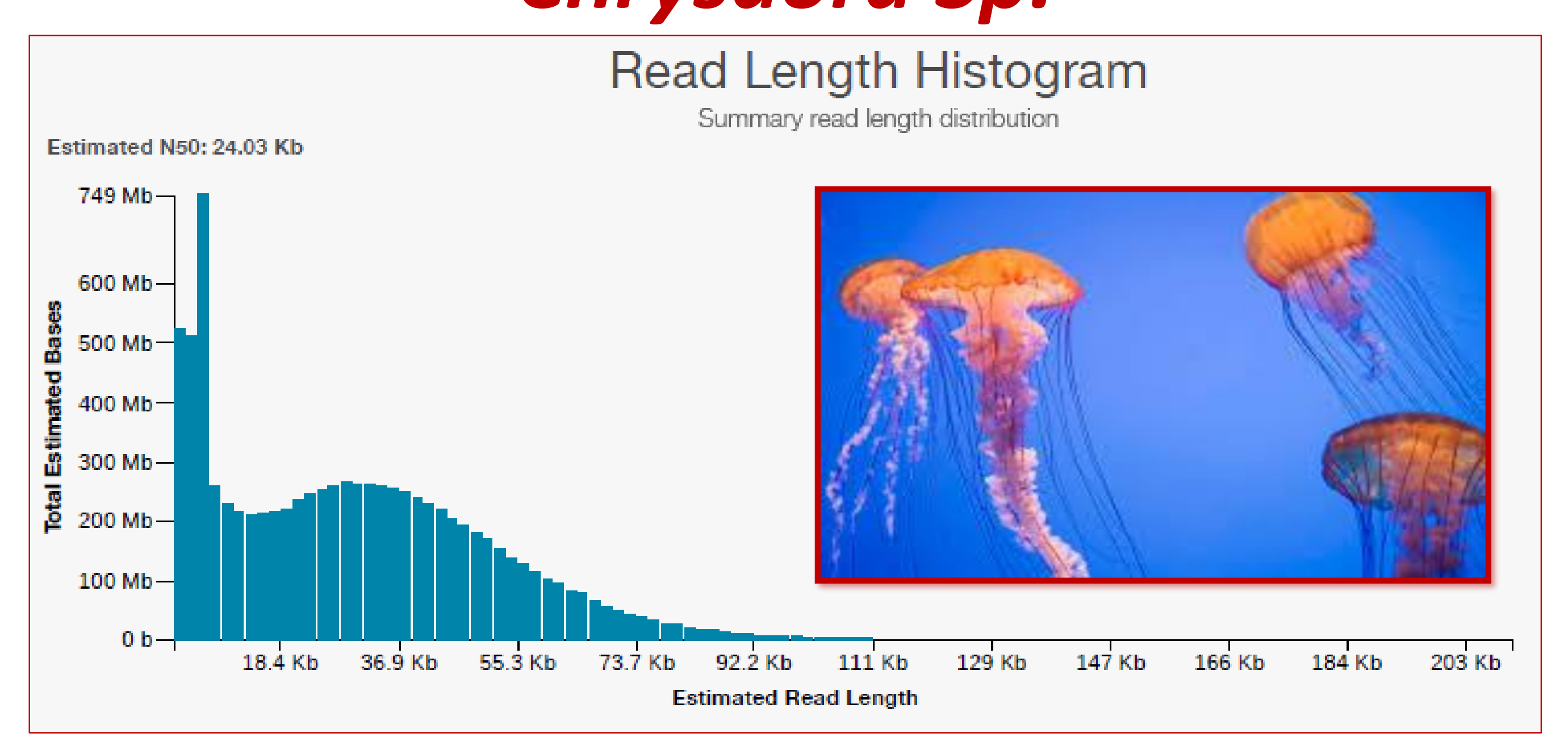

Assessment of our bespoke protocol on other different marine species

\section{Conclusion}

In this work, we investigated different protocols to extract DNA from marine species in order to achieve long read sequences using ONT while attempting to utilize the full flow cell capacity. None of the frequently used treatments were satisfactory. A new protocol was thus concocted from the combination of different steps of the common methods. When applying the optimized protocol to other marine species, we observed increase in the flow-cell capacity during the run, from $54 \%$ to more than $86 \%$ reaching sometimes to $92 \%$, with a total of $10 \mathrm{~Gb}$ data, instead of 1 to $2 \mathrm{~Gb}$, usually generated by a single flowcell within 1-day sequencing run. Using the established protocol, we were able to get rid of the inhibition that is frequently observed when sequencing marine species. Furthermore, it allowed us to obtain high-quality/high molecular weight DNA that enabled the generation of long reads sequences required for whole genome assembly of the potential Qatari new species. 